\title{
A Model Approach for Binder Selection in Binder Jetting
}

Sergio I. Yanez-Sanchez* ${ }^{1}$, Martin D. Lennox ${ }^{1}$, Daniel Therriault ${ }^{2}$, Basil D. Favis ${ }^{1}$, Jason R.

$$
\text { Tavares }^{* 1}
$$

${ }^{1}$ CREPEC, Department of Chemical Engineering, Polytechnique Montreal, Montréal, QC, H4T

$$
\text { 1J4, Canada }
$$

${ }^{2}$ CREPEC, Department of Mechanical Engineering, Polytechnique Montreal, Montréal, QC, H4T 1J4, Canada

\section{S1. Powder material details}

Scanning electron microscopy (SEM) Backscattered electrons (BSE) images of the powder can

be observed in Figure S1 and Table S1 contains its composition.
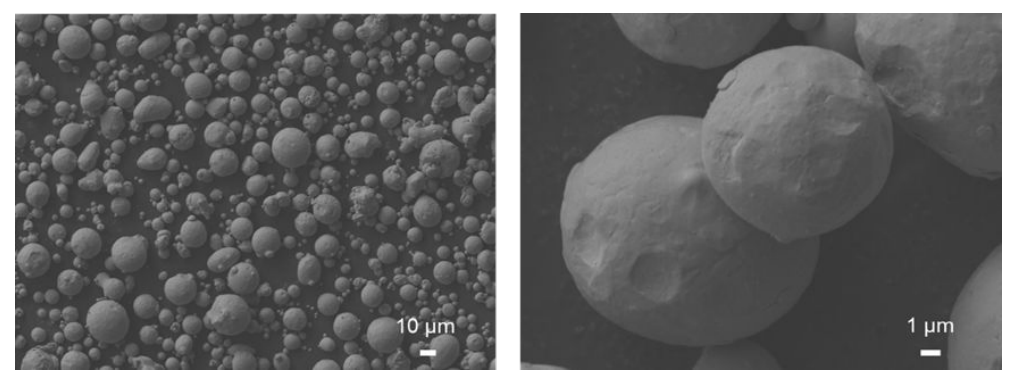

Figure S1. BSE SEM images of the SS316L powder. 
Table S1. Composition of the SS 316L powder.

\begin{tabular}{ccccccccccc}
\hline Element & $\mathbf{C r}$ & $\mathbf{N i}$ & $\mathbf{M o}$ & $\mathbf{M n}$ & $\mathbf{N}$ & $\mathbf{S i}$ & $\mathbf{C}$ & $\mathbf{P}$ & $\mathbf{O}$ & $\mathrm{Fe}$ \\
\hline At. \% & 17.13 & 10.22 & 2.42 & 1.28 & 0.1 & 0.22 & 0.03 & 0.02 & 0.075 & Bal. \\
\hline
\end{tabular}

\section{S2. Binder distribution}

The binder saturation ratio of the hand-made green bodies was slightly higher than $100 \%$. In fact,

the samples presented a sedimentation effect: the volume of binder exceeded that of the space between the powder particles. This caused the particles to sink and the excess liquid to accumulate at the top of the piece. This effect was only apparent under a microscope. Figure S2 illustrates this effect. It presents SEM images of different parts of the same sample. One can see that the bottom and middle have similar binder distribution (seen as dark areas) whereas the top part is filled by polymer. This means that there is an accumulation of binder on the top of the sample. However, compared to the height of the pieces $(10 \mathrm{~cm})$, this occurs in a very thin layer $(\sim 50 \mu \mathrm{m})$. Thus, the effect of this binder accumulation is considered negligible and is assumed not to have a significant impact on the results. 

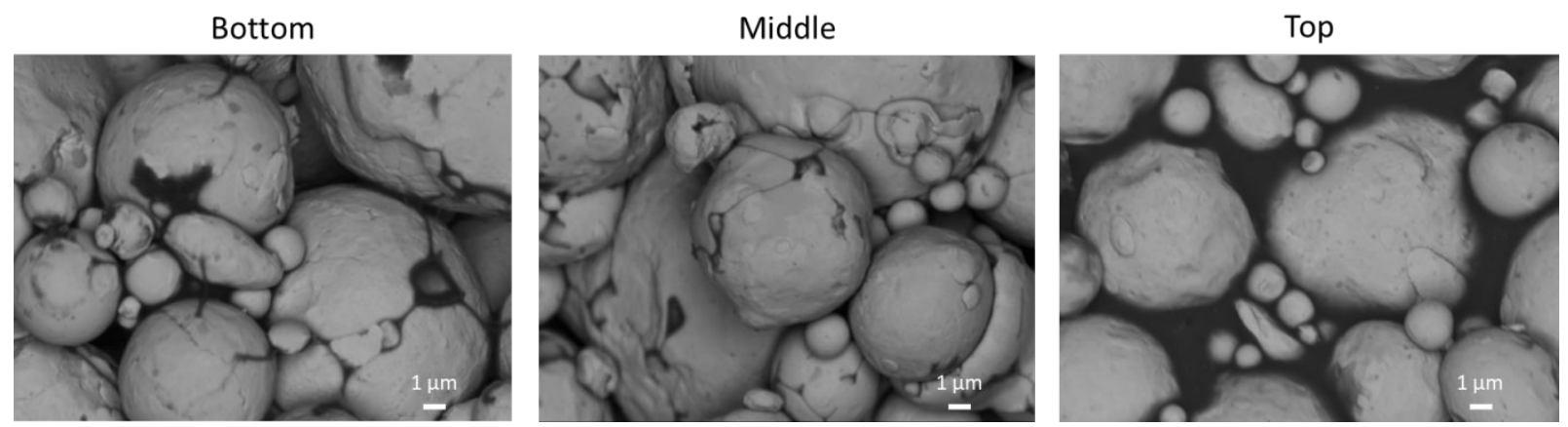

Figure S2. Binder distribution on a green body with respect to the height of the piece $\left(C_{g}=0.69\right.$

wt. \%). The three images come from different broken parts of the same sample.

\section{S3. Interparticle bridge length measurement}

ImageJ is an image processing open-source software. It was used to measure the length of the

interparticle bridges on SEM BSE images. Six bridges were measured, and every bridge was

measured 6 times. Figure S3 shows an example of the measurement. It is to be noted that the

transition from places where the binder is present to spaces where there is no binder are difficult to

find. However, there are spaces where one can be certain of the presence of binder. In Figure S3,

the yellow arrows point at regions with clear presence of polymer while the white arrows point at

regions where the presence of polymer is not evident. 


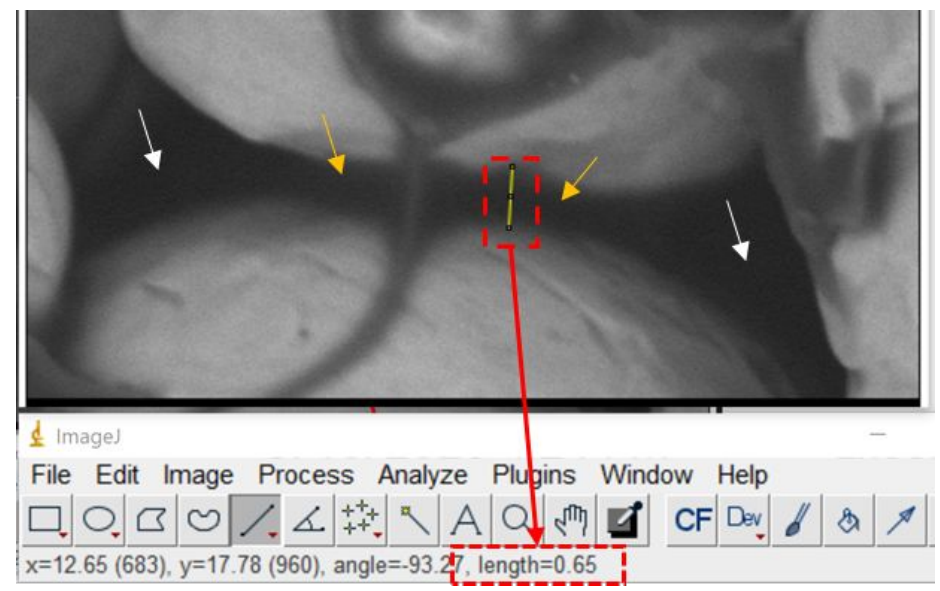

Figure S3. Bond length measurement with ImageJ. The white arrows point at spaces that are probably. The yellow arrow point at the limits of the binder bridge distinguishable with the eye. 